\title{
Recent Drug Resistant Epilepsy Spectrum in Eastern India
}

Original Article

Journal of Epilepsy Research pISSN 2233-6249 / eISSN 2233-6257

Received December 3, 2016 Accepted March 4, 2017

Corresponding author: Joydeep Mukherjee Department of Neurology, NRS Medical College Hospital, 33 Sarat Bose Road, PO-Rajbari Colony, PIN-700081, India. Tel. +91-983-651-0228

E-mail; joydeepdoc@gmail.com

\section{Joydeep Mukherjee, Durga Prasad Chakraborty, Gautam Guha, Biman Bose, Shankar Prasad Saha} Department of Neurology, NRS Medical College Hospital, Kolkata, India

Background and Purpose: The Magnitude of Drug-resistant Epilepsy (DRE) in India, being unknown, takes a heavy toll on the patients and society in the form of prolonged dependence, unemployment, morbidity and mortality. We tried to explore the clinical, electro-physiological, neuro-imaging and drug-response spectrum of DRE patients in Eastern India in our study.

Methods: During the period of January 2014 to December 2015, epilepsy patients were treated and DRE patients were identified according to International League Against Epilepsy criteria. We isolated those patients and studied them in a special clinic.

Results: Among 2,153 patients treated in Neurology out-patient department, 243 (11.3\%) patients were drug-resistant. Among the DRE patients, $63 \%$ were male. Age-wise 40\%, 30.5\% \& $18.1 \%$ patients were presented in their first, second and third decades respectively. Males were more affected in 0-5 years age group while females in 6-10 years age group. Various seizures types were found alone or in combination. Males were mostly affected by generalized tonic clonic seizure and myoclonus and females by complex partial seizure. Positive family history was higher in partial seizure group. Electroencephalographic (EEG) abnormalities were common with structural lesions in brain. EEG findings in different etiologies were varied with a large number of DRE patients who were found to have normal EEG. Females were higher medicine non-compliant.

Conclusions: The spectrum was pointed towards gender predilection for specific age group and also for seizure types. Idiopathic cases were most common in DRE, pointing towards the need of newer investigations. Normal EEG could be found even in a DRE patient. Non-compliance was more in females.

(2017;7:39-44)

Key words: Drug resistant epilepsy, Developing countries, Electroencephalogram, Gender, Eastern India

\section{Introduction}

Epilepsy is a disease of the brain defined by any of the following conditions: 1) At least two unprovoked (or reflex) seizures occurring $>24 \mathrm{~h}$ apart, 2) One unprovoked (or reflex) seizure and a probability of further seizures similar to the general recurrence risk (at least $60 \%$ ) after two unprovoked seizures, occurring over the next 10 years, 3) Diagnosis of an epilepsy syndrome. ${ }^{1}$

Epilepsy is one of the most common neurological disorders. India is home of about 10 million people with epilepsy, 2.47 to 8 per 1,000 population in rural ${ }^{2-14}$ and 2 to 7.8 per 1,000 population in urban areas. $^{2,15-24}$ Incidence was varied from 0.27 to 0.6 per 1,000 population per year in different parts of India. ${ }^{13,17,25,26}$ Drug resistant epilepsy was defined by International League Against Epilepsy (ILAE) ${ }^{27}$ as failure of adequate trials of two tolerated and appropriately chosen and used anti-epileptic drug (AED) schedules (whether as mono-therapies or in combination) to achieve sustained seizure freedom. Seizure freedom is defined as freedom from seizures for a minimum of three times the longest pre-intervention inter-seizure interval (determined from seizures occurring within the past 12 months) or 12 months, whichever is longer. On the other hand, treatment failure is defined as recurrent seizure(s) after the intervention has been adequately applied (as defined earlier). If a patient has been seizure-free for three times the pre-intervention inter-seizure interval but for $<12$ months, seizure control should be categorized as "undetermined". The prevalence and incidence of drug resistant epilepsy (DRE) in India was unknown. Result was varied among studies abroad, a study in Spain showed $19 \%{ }^{28}$ and a study in Thailand 
showed $42 \%{ }^{29}$ epilepsy patients were drug resistant. DRE patients and their families were suffering the most. Depression, lower education level, and unemployment were playing heavy toll on them. ${ }^{30}$ So we took up the present study as a global approach to DRE patients in Eastern India.

\section{Methods}

We conducted our study in the Neurology department of Nilratan Sircar Medical College and Hospital in Eastern India, which serves patients from the states of Eastern India namely, West Bengal, Bihar, Jharkhand, Odisha and Sikkim as well as from countries such as Bangladesh and Nepal. We took them in a separate Intractable Epilepsy Clinic attached with our department and studied them. Apart from demographic parameters, detailed seizure history was noted. Electroencephalogram (EEG) was done on all patients. Every patient was investigated with structural imaging magnetic resonance imaging (MRI) of brain to rule out/confirm structural pathology and divided into primary/idiopathic and secondary. Idiopathic/ primary DRE were the patients with normal brain imaging where no cause could be detected on MRI scan; others were cases with abnormal brain imaging. However normal MRI brain does not establish it to be primary as many secondary cases may show normal MRI brain. Functional imaging and other sophisticated investigations could have found the etiology. Detailed treatment history was noted along with drug compliance. Non-compliance was defined as very irregular intake of AED as well as frequent drop-outs. Statistical analysis was done with Instat GraphPad (GraphPad Software Inc., CA, USA) using Fisher's exact test and two sided $p$ values were measured.

\section{Results}

From a total of 22,638 patients who attended Neurology out-patient department (OPD) from January 2014 to December 2015, we detected and treated $2153(9.5 \%)$ epilepsy patients. Among those epilepsy patients, $243(11.3 \%)$ patients were found to be suffering from DRE according to the guideline of ILAE. ${ }^{27}$

We found male preponderance (63\%) in our series of total 243 DRE patients (Table 1). Most of the patients (40\%) were presented in their first decade of life whereas $30.5 \%$ patients were presented in their second decade and $18.1 \%$ in their third decade (Fig. 1).Gender bias was also found in the study. In the 0-5 year age group, males were affected significantly higher than their female counterparts
( $18.8 \%$ vs. $9 \%, p=0.04$ ) but in the $6-10$ years age group, females were affected significantly higher than their male counterparts $(19.5 \%$ vs. $33.7 \%, p=0.02)$ (Fig. 1). Various seizure types were noted in different patients. Some patients were suffering from only single seizure type, but others were suffering from multiple of seizure types. Correlation of seizure type with gender was also looked for. Males were having predominantly generalized tonic clonic seizure (GTCS) \& Myoclonic seizure, whereas complex partial seizure was dominated in females (Table 2).

One hundred and twenty-nine male and seventy-seven female patients were suffering from single seizure type but twenty-four male and thirteen female patients were suffering from multiple seizure types; there were no significant difference between genders ( $p=$ 0.85 ) (Fig. 2). Family history of seizure disorder was present in $11.5 \%$ DRE patients; which was higher in partial seizure patients than generalized seizure patients ( $16.6 \%$ vs. $5.2 \%, p=0.07$ ), the statistical difference being insignificant.

Most of the patients were suffering from primary/ idiopathic resistant epilepsy $(n=168)$ followed by Hypoxic Ischemic Encephalop-

Table 1. Characteristics of DRE patients

\begin{tabular}{lc}
\hline Characteristics of drug resistant epilepsy patients & Values \\
\hline Median age & 12.7 \\
Male & $63 \%$ \\
Female & $37 \%$ \\
Percentage of focal epilepsy & $46.1 \%$ \\
Percentage of generalized epilepsy & $38.7 \%$ \\
Percentage of multiple seizure type & $15.2 \%$ \\
Presence of family history & $11.5 \%$ \\
Mean number of AED use & 2.36 \\
Medicine compliance & $72.4 \%$ \\
\hline DRE, drug resistant epilepsy; AED, anti-epileptic drug.
\end{tabular}

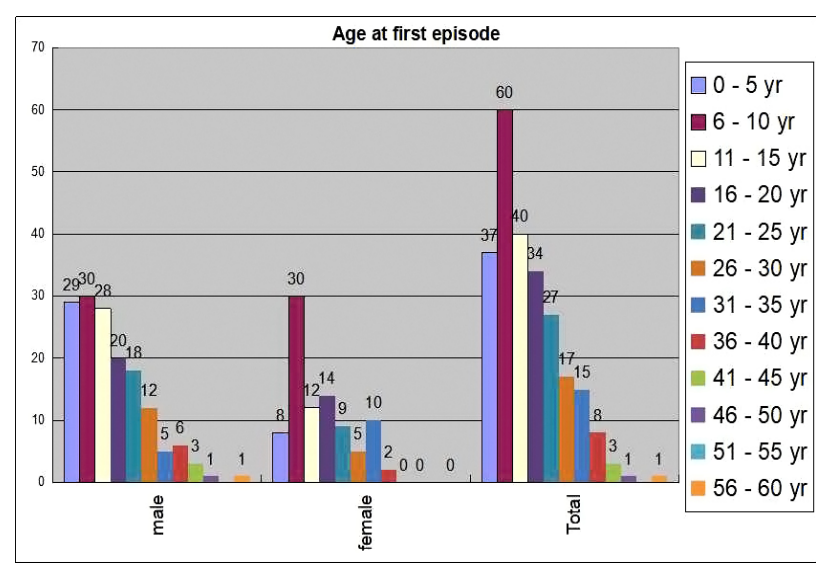

Figure 1. Pattern of age first episode of epilepsy in male, female and total. yr, year. 
Table 2. Gender predilection in GTCS, Myoclonus and CPS

\begin{tabular}{lccc}
\hline & Male & Female & $p$ value \\
\hline GTCS alone & 51 & 22 & \multirow{2}{*}{0.06} \\
CPS alone & 35 & 30 & \\
\hline Myoclonus alone & 14 & 2 & 0 \\
CPS alone & 35 & 30 & 0.02 \\
\hline
\end{tabular}

GTCS, generalized tonic clonic seizure; CPS, complex partial seizure.

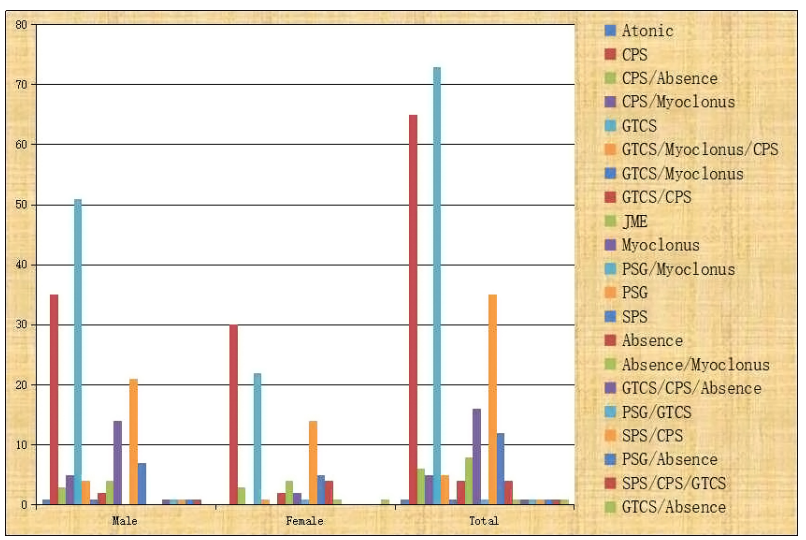

Figure 2. Pattern of various seizure types in male, female and total patients.

athy $(n=13)$, granuloma $(n=13)$ and hippocampal Sclerosis $(n=11)$ and others (Fig. 3). EEG findings were varied in different resistant epilepsy etiologies. Normal EEG was most prevalent in Primary and Hippocampal sclerosis cases; generalized discharge was most prevalent in Granuloma, Hypoxic ischemic encephalopathy and Gliosis; Focal discharge was most prevalent in congenital malformation cases (Table 3). It was found that, EEG abnormalities were found in higher percentage in resistant epilepsy cases with abnormal brain imaging (secondary resistant epilepsy cases), than resistant epilepsy cases with normal brain imaging (primary/idiopathic resistant epilepsy cases) $(63.4 \%$ vs. $58.3 \% ; p=0.02$ ) (Table 4).

We used phenytoin, sodium valproate, carbamazepine or phenobarbitone as the first AED according to the epilepsy type. Regarding the second drug choice, we found that, carbamazepine was used in highest number (18.1\%), only to be followed by clobazam and levetiracetam (16.2\% each) (Fig. 4). At the time of data collection, 18 (7.4\%) patients were on four AEDs, 85 (35\%) patients were on three AED, 107 (44\%) patients were on two AED and 33 (13.6\%) patients were on one AED. Two hundred and ten patients were taking two or more drugs. Females were significantly higher medicine non-compliant (34.4\%) compared to male $(23.5 \%)(p<0.0001)$.

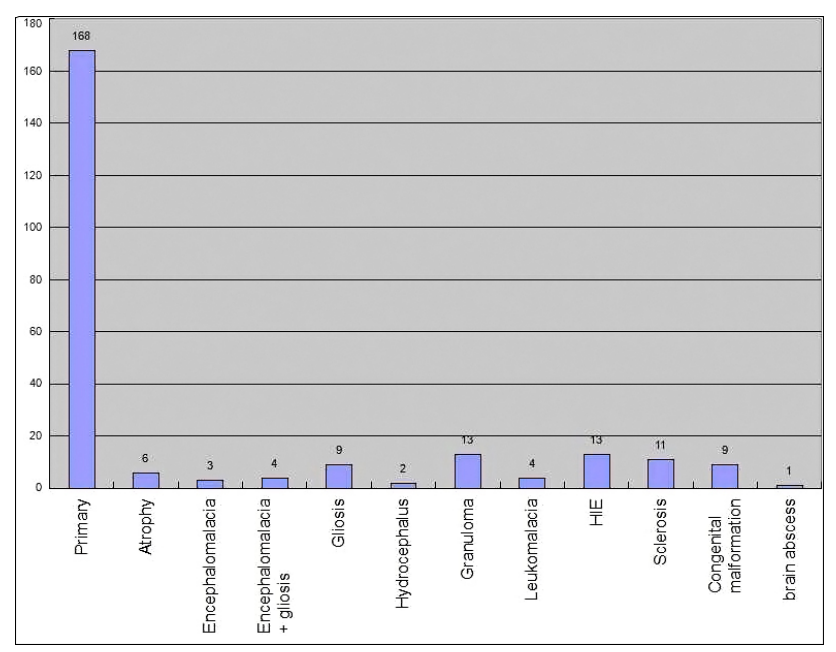

Figure 3. Different etiologies in drug resistant epilepsy (DRE) patients.

\section{Discussion}

It was quite obvious from our day to day clinical experience, that a high number of epilepsy patients were drug resistant. Lagunju et al. ${ }^{31}$ in Nigeria found 17.2\%, Ramos et al. ${ }^{28}$ in Spain found 19\%, Berg et al. ${ }^{32}$ in United States of America found $23.2 \%$, Kwan et al. ${ }^{33}$ in Scotland found $37 \%$ drug resistant in their epilepsy patients. Boonluksiri et al. ${ }^{29}$ found lesser intractability (23.4\%) in focal seizure patients in Thailand. Tripathi et al. ${ }^{34}$ in North India compared intractable and controlled seizure patients. In their Intractable group, they found partial seizures in $83 \%$ patients, generalized seizure in $7 \%$ patients, myoclonic seizure in $6.5 \%$ patients and multiple seizure types in $3.5 \%$ patients. Whereas, in their well controlled group, $56.5 \% \mathrm{pa}-$ tients were presented with partial seizures, 30.5\% patients were presented with generalized seizure, and $12.5 \%$ patients were presented with myoclonic seizures as the initial presentation. We found 11.3\% DRE cases from our total epilepsy cohort. This variability might be due to genetic variation of different regions of the world; however, socio-economic differences might play a role in using newer generation costly AEDs, thus reducing the number of DRE cases.

Ramos et al. ${ }^{28}$ found $59 \%$ males in their population of DRE cases, whereas Tripathi $\mathrm{M}$ et al from North-India found as high as 71\% male in their DRE series ${ }^{34}$. All recent studies on epilepsy from many parts of India showed male predominance ${ }^{13,16-18,20,21,35}$ except the study done by Pandey et al. ${ }^{36}$ in Chandigarh, North India where females were mostly reported. Wirrell et al. ${ }^{37}$ found male preponderance $(51 \%)$ in their DRE series. We found male patients comprising 
Table 3. Spectrum of various EEG findings in different etiology of DRE patients

\begin{tabular}{lcccccc}
\hline EEG Findings & $\begin{array}{c}\text { Primary } \\
(\mathrm{n}=168)\end{array}$ & $\begin{array}{c}\text { Granuloma } \\
(\mathrm{n}=13)\end{array}$ & $\begin{array}{c}\text { Hypoxic Ischemic } \\
\text { Encephalopathy } \\
(\mathrm{n}=13)\end{array}$ & $\begin{array}{c}\text { Hippocampal } \\
\text { Sclerosis } \\
(\mathrm{n}=11)\end{array}$ & $\begin{array}{c}\text { Gliosis } \\
(\mathrm{n}=9)\end{array}$ & $\begin{array}{c}\text { Congenital } \\
\text { malformation } \\
(\mathrm{n}=9)\end{array}$ \\
\hline Normal & 42 & 46 & 24 & 82 & 33 & 22 \\
Generalized discharge & 39 & 54 & 46 & 18 & 67 & 22 \\
Focal discharge & 8 & 0 & 15 & 0 & 0 & 56 \\
Focal with secondary & 11 & 0 & 15 & 0 & 0 & 0 \\
\hline generalization & 11 & & & 0 & \\
\hline
\end{tabular}

Values are presented as number (\%).

EEG, electroencephalography; DRE, drug sensitive epilepsy.

Table 4. Comparison of different EEG discharges between patients with and without structural brain lesions

\begin{tabular}{lcc}
\hline EEG Findings & $\begin{array}{c}\text { Cases without } \\
\text { structural brain } \\
\text { lesion (primary) } \\
(\mathrm{n}=168)\end{array}$ & $\begin{array}{c}\text { Cases with } \\
\text { structural brain } \\
\text { lesion (secondary) } \\
(\mathrm{n}=75)\end{array}$ \\
\hline Normal & 41.7 & 36.6 \\
Generalized discharge & 38.9 & 46.3 \\
Focal discharge & 8.3 & 12.2 \\
Focal with secondary & 11.1 & 4.9 \\
$\quad$ generalization & & \\
\hline
\end{tabular}

Values are presented as number (\%).

EEG, electroencephalography.

$63 \%$ of our study population. Higher male epilepsy cases might be due to higher family care for male patients but higher male DRE cases would raise the suspicion that males would have some attribute to get drug resistant, to be explored in future trials.

Ramos et al. ${ }^{28}$ found significantly high (63\%) percentage of DRE cases in $<4$ year age group and 37\% in 4-13 year group in a childhood DRE series among epilepsy patients and Tripathi et al..$^{34}$ found majority of the intractable cases had age at onset below 14 years. Most of the patients were presented in their first decade of life in our study which was well corroborated to similar studies. The finding was pointed to some attributes, might be inheritable or congenital in nature which affected the brain at early age to render the case DRE. Wirrell et al. ${ }^{37}$ studied for the predictors and course of DRE in patients who had onset before 36 months of age and found that Medical intractability was most commonly seen early: $61 \%$ met criteria by the end of the first year, $86 \%$ by the end of the third year, and $93 \%$ by the end of the fifth year after initial diagnosis of epilepsy. Intractability after 1 year was similar in children with focal onset $(21 / 33,63.6 \%)$ and generalized seizures/spasms (8/11, 72.7\%). Of those with focal seizures, $90.9 \%$ met criteria for intractability by the third year after diagnosis.

Christensen et al. ${ }^{38}$ found more females than males, who were diagnosed with idiopathic generalized epilepsy. Overall, no gender dif-

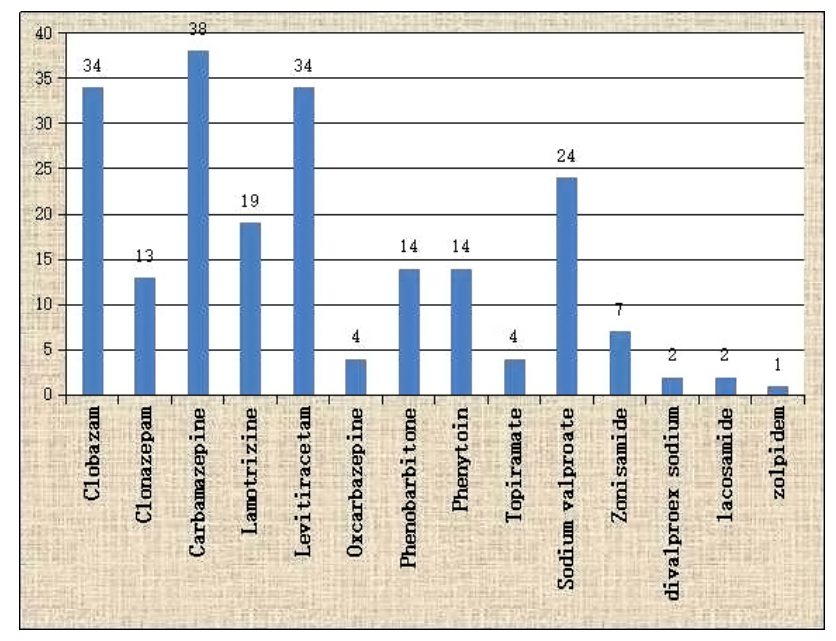

Figure 4. Pattern of first add-on drug in drug resistant epilepsy (DRE) patients.

ference was found in localization related epilepsy, but localization-related symptomatic epilepsies were more frequent in males, and cryptogenic localization-related epilepsies were more frequent in females. GTCS and Myoclonic seizure were more common in male and focal seizure was more common in female in our study. No specific reason was found even after a vast literature search.

Baraitser ${ }^{39}$ found $4-10 \%$ increased risk of epilepsy in patients with positive family history with 1-3 close family members accordingly. We found $11.5 \%$ DRE cases had positive family history of epilepsy, but the reason behind higher prevalence in partial seizure cases was not known.

Ramos et al. ${ }^{28}$ studied drug resistant epilepsy for testing new ILAE criteria for the same. They found that $42 \%$ of total epilepsy patients and $60 \%$ in DRE patients having structural abnormalities in brain. Tripathi et al. ${ }^{34}$ while searching for predictors of drug resistant epilepsy in North India in a case-control study, found that Abnormal brain imaging was seen in $79 \%$ of the patients in intractable group and only $39 \%$ of the patients in well control group. In our study, 69.1\% DRE cases did not have any structural abnormality in brain 
(Fig. 3). That finding had pointed on the need of functional imaging of brain in similar patients. A few cases with structural abnormalities in brain might need surgery for remission.

Variety of different EEG finding in our study was depicted in Table 3 and comparison between cases with and without structural brain lesion was done in Table 4. Boonluksiri et al. ${ }^{29}$ described abnormal EEG in 22.3\% in their cohort of DRE + DSE patients. We found a significantly high number of drug resistant epilepsy cases having normal EEG. The number was high (82\%) in hippocampal sclerosis and low (22\%) in congenital malformation (Table 3). The origin and spread of the epileptic current might be in deeper brain tissue to be detected in surface EEG in those cases. The finding was a significant concern for general practitioners so that, they should not exclude epilepsy even if the surface EEG was found to be normal, rather should have suspicion that the case might turn into drug-resistant.

Carbamazepine was used mostly as the second drug, to be followed by clobazam and levetiracetam and continued successfully. Government has free supply of phenytoin, carbamazepine, sodium valproate and phenobarbitone. Patients collect the free medicines from hospital after check-up in OPD. There is a limit of providing medicine for one month after each check-up. Other AED could be bought from 'fair price shop' in subsidized rate. Free supply from Government clinic might be the first cause for highest use of carbamazepine, to be followed by cheap price, wide availability, good tolerance and efficacy. However, clobazam and levetiracetam might got its high position due to high efficacy and tolerance; though costlier and not supplied from government hospital.

We found drug compliance was significantly lesser in females than their male counterparts. Significantly lesser health concern for females in developing countries was the most important cause, as males were predominantly the bread-earners for the families.

It is a small endeavor from our part, to study the prevalence of DRE in this part of the country which may or may not be at par with the other parts of the country. In future, if there is any such study in other parts of the country, we can compare the data with the other part. So far the ethnicity and clinical practice are concerned, there is not much difference with the other parts of the country. Our Institute caters a huge population of Eastern India and the countries such as Bangladesh and Nepal. In our opinion, this study aims at showing a total picture of this region.

In conclusion, this study shows predilection of different age groups in childhood to different genders. We also found specific seizure types having gender predilection. Family history had a role in
DRE. Idiopathic was the most common cause of DRE. EEG finding were varied in different etiologies. Normal EEG never excluded DRE. Females were higher medicine non-compliant.

\section{Acknowledgments}

We acknowledge our sincere gratitude to Dr. Atri Chatterjee, Dr. Bhabesh Solanki, Dr. Pragateshnu Das, Dr. Uma SinhaRoy, Dr. Bhaskar Brojobasi, Dr. Soumava Mukherjee, Dr. Nityananda Tikadar and Dr. Susanta Kumar Bar for their active co-operation and support during the study.

\section{References}

1. Fisher RS, Acevedo C, Arzimanoglou A, et al. ILAE official report: a practical clinical definition of epilepsy. Epilepsia 2014;55:475-82.

2. Sridharan R, Murthy BN. Prevalence and pattern of epilepsy in India. Epilepsia 1999;40:631-6.

3. Goel D, Agarwal A, Dhanai JS, et al. Comprehensive rural epilepsy surveillance programme in Uttarakhand state of India. Neurol India 2009:57:355-6.

4. Sureka RK, Sureka R. Prevalence of epilepsy in rural Rajasthan-a door-to-door survey. J Assoc Physicians India 2007;55:741-2.

5. Murthy JM, Vijay S, Ravi Raju C, et al. Acute symptomatic seizures associated with neurocysticercosis: a community-based prevalence study and comprehensive rural epilepsy study in South India (CRESSI). Neurol Asia 2004;9:86.

6. Saha SP, Bhattacharya S, Das SK, et al. Epidemiological study of neurological disorders in a rural population of Eastern India. J Indian Med Assoc 2003;101:299-300, 302-4.

7. Kokkat AJ, Verma AK. Prevalence of seizures and paralysis in a rural community. J Indian Med Assoc 1998;96:43-5.

8. Shaji S, Verghese A, Promodu K, et al. Prevalence of priority psychiatric disorders in a rural area in kerala. Indian J Psychiatry 1995;37:91-6.

9. Premarajan KC, Danabalan M, Chandrasekar $R$, et al. Prevalence of psychiatry morbidity in an urban community of Pondicherry. Indian J Psychiatry 1993;35:99-102.

10. Kapoor S, Banerjee A. Prevalence of common neurological diseases in a rural community of India. Indian J Community Med 1989;14:171.

11. Koul R, Razdan S, Motta A. Prevalence and pattern of epilepsy (Lath/Mirgi/Laran) in rural Kashmir, India. Epilepsia 1988;29:116-22.

12. Das S, Sanyal K. Neuroepidemiology of major neurological disorders in rural Bengal. Neurol India 1996;44:47.

13. Mani KS, Rangan G, Srinivas $H V$, et al. The Yelandur study: a community-based approach to epilepsy in rural South India--epidemiological aspects. Seizure 1998;7:281-8. 
14. Pal DK, Das T, Sengupta S. Comparison of key informant and survey methods for ascertainment of childhood epilepsy in West Bengal, India. Int J Epidemio/ 1998;27:672-6.

15. Singh G, Bawa J, Chinna D, et al. Association between epilepsy and cysticercosis and toxocariasis: a population-based case-control study in a slum in India. Epilepsia 2012;53:2203-8.

16. Raina SK, Razdan S, Nanda R. Prevalence of neurological disorders in children less than 10 years of age in RS Pura town of Jammu and Kashmir. J Pediatr Neurosci 2011;6:103-5.

17. Banerjee TK, Ray BK, Das SK, et al. A longitudinal study of epilepsy in Kolkata, India. Epilepsia 2010;51:2384-91.

18. Banerjee TK, Hazra A, Biswas A, et al. Neurological disorders in children and adolescents. Indian J Pediatr 2009;76:139-46.

19. Das SK, Biswas A, Roy J, et al. Prevalence of major neurological disorders among geriatric population in the metropolitan city of Kolkata. J Assoc Physicians India 2008;56:175-81.

20. Das SK, Biswas A, Roy $T$, et al. A random sample survey for prevalence of major neurological disorders in Kolkata. Indian J Med Res 2006;124:163-72.

21. Radhakrishnan K, Pandian JD, Santhoshkumar T, et al. Prevalence, knowledge, attitude, and practice of epilepsy in Kerala, South India. Epilepsia 2000;41:1027-35.

22. Gourie-Devi M, Gururaj G, Satishchandra P, et al. Neuro-epidemiological pilot survey of an urban population in a developing country. A study in Bangalore, south India. Neuroepidemiology 1996;15:313-20.

23. Sen B, Nandi DN, Mukherjee SP, Mishra DC, Banerjee G, Sarkar S. Psychiatric morbidity in an urban slum-dwelling community. Indian J Psychiatry 1984;26:185-93.

24. Bharucha NE, Bharucha EP, Bharucha $A E$, et al. Prevalence of epilepsy in the Parsi community of Bombay. Epilepsia 1988;29:111-5.

25. Saha SP, Bhattachrya S, Roy BK, et al. A prospective incidence study of epilepsy in a rural community of West-Bengal, India. Neurol Asia 2008; 13:41-8.

26. Sawhney IM, Singh A, Kaur $P$, et al. A case control study and one year follow-up of registered epilepsy cases in a resettlement colony of North
India, a developing tropical country. J Neurol Sci 1999;165:31-5.

27. Kwan P, Arzimanoglou A, Berg AT, et al. Definition of drug resistant epilepsy: Consensus proposal by the ad hoc Task Force of the ILAE Commission on Therapeutic Strategies. Epilepsia 2010:51:1069-77.

28. Ramos $\amalg$, Rodriguez $L M I$, Angiler $L P$, et al. A study of drug-resistant epilepsy testing the new ILAE criteria. Seizure 2012:266-72.

29. Boonluksiri $P$, Visuthibhan A, Katanyuwong K. Clinical prediction rule of drug resistant epilepsy in children. J Epilepsy Res 2015;5:84-8.

30. Akdemir V, Sut $N$, Guldiken B. Factors affecting the quality of life in drug-resistant epilepsy patients. Acta Neurol Belg 2016;116:513-8.

31. Lagunju IA, Asinobi A. Predictors of early seizure remission in Nigerian children with newly diagnosed epilepsy. Afr J Med Med Sci 2011;40:239-45.

32. Berg AT, Vickrey BG, Testa FM, et al. How long does it take for epilepsy to become intractable? A prospective investigation. Ann Neurol 2006;60:73-9.

33. Kwan P, Brodie MJ. Early identification of refractory epilepsy. N Engl J Med 2000;342:314-9.

34. Tripathi M, Padhy UP, Vibha $D$, et al. Predictors of refractory epilepsy in north India: a case-control study. Seizure 2011;20:779-83.

35. Shah PA, Shapoo SF, Koul RK, et al. Prevalence of epilepsy in school-going children (6-18 years) in Kashmir valley of north-west India. J Indian Med Assoc 2009;107:216-8.

36. Pandey $S$, Singhi $P$, Bharti B. Prevalence and treatment gap in childhood epilepsy in a north Indian city: a community-based study. J Trop Pediatr 2014;60:118-23.

37. Wirrell E, Wong-Kisiel L, Mandrekar J, et al. Predictors and course of medically intractable epilepsy in young children presenting before 36 months of age: a retrospective, population-based study. Epilepsia 2012:53:1563-9.

38. Christensen J, Kjeldsen MJ, Andersen $H$, et al. Gender differences in epilepsy. Epilepsia 2005;46:956-60.

39. Baraitser M. Relevance of a family history of seizures. Arch Dis Child 1983;58:404-5. 